J. Korean Math. Soc. 49 (2012), No. 4, pp. 829-842

http://dx.doi.org/10.4134/JKMS.2012.49.4.829

\title{
BOUNDEDNESS OF BEREZIN TRANSFORM ON HERZ SPACES
}

\author{
Chu-Hee Cho and Kyunguk Na
}

\begin{abstract}
In this paper, we give the condition for the boundedness of the Berezin transforms on Herz spaces with a normal weight on the unit ball of $\mathbb{C}^{n}$. And we provide the integral estimates concerning pluriharmonic kernel functions. Using this, we finally obtain the growth estimates of the Berezin transforms on such Herz spaces.
\end{abstract}

\section{Introduction}

For $n \geq 2$, let $B=B_{n}$ be the open unit ball in $\mathbb{C}^{n}$. Given $\alpha>-1$ and $1 \leq p \leq \infty$, let $L_{\alpha}^{p}=L^{p}\left(V_{\alpha}\right)$ be the weighted Lebesgue space which consists of all complex-valued functions $f$ on $B$ such that

$$
\|f\|_{L_{\alpha}^{p}}=\left\{\int_{B}|f|^{p} d V_{\alpha}\right\}^{1 / p}<\infty,
$$

where $V$ denotes the Lebesgue volume measure on $B$ and $d V_{\alpha}(z)=(1-$ $\left.|z|^{2}\right)^{\alpha} d V(z)$ is a measure with a normal weight. The weighted pluriharmonic Bergman space $b_{\alpha}^{2}=b_{\alpha}^{2}(B)$ is the set of all pluriharmonic functions $f$ on $B$ with $f \in L_{\alpha}^{2}$. We also write $R_{z}^{\alpha}(w)=R^{\alpha}(z, w)$ be the reproducing kernel for $b_{\alpha}^{2}$. It is known that

$$
b_{\alpha}^{2}=A_{\alpha}^{2}+\overline{A_{\alpha}^{2}},
$$

where $A_{\alpha}^{2}=A_{\alpha}^{2}(B)$ is the holomorphic Bergman space with respect to the normal weight $\left(1-|z|^{2}\right)^{\alpha}$; see [4] for details. Thus, we see that the explicit formula of $R_{z}^{\alpha}(w)$ is as follows:

$$
R_{z}^{\alpha}(w)=\frac{1}{(1-\bar{z} \cdot w)^{n+\alpha+1}}+\frac{1}{(1-z \cdot \bar{w})^{n+\alpha+1}}-1
$$

Received May 21, 2011; Revised October 21, 2011.

2010 Mathematics Subject Classification. Primary 31B10.

Key words and phrases. Berezin transform, Schatten space, Herz space.

The first author was supported by Basic Science Research Program through the National Research Foundation of Korea(NRF) grant funded by the Korea government(MEST)(20110001251).

The second author was supported by Hanshin University Research Grant. 
for $z, w \in B$. Here and subsequently, $z \cdot \bar{w}=z_{1} \bar{w}_{1}+\cdots+z_{n} \bar{w}_{n}$ denotes the Hermitian inner product on $\mathbb{C}^{n}$.

It is easy to obtain the following estimates from (1.1):

$$
R_{z}^{\alpha}(z) \approx \frac{1}{\left(1-|z|^{2}\right)^{n+\alpha+1}} \quad \text { and } \quad\left|R_{z}^{\alpha}(w)\right| \lesssim \frac{1}{|1-z \cdot \bar{w}|^{n+\alpha+1}}
$$

for all $z, w \in B$, so $R_{z}^{\alpha}$ is bounded for fixed $z \in B$.

Given a positive (finite) Borel measure $\mu$ on $B$ (we will write $\mu \geq 0$ for simplicity), the Berezin transform is defined by

$$
\widetilde{\mu}(z)=\frac{1}{R_{z}^{\alpha}(z)} \int_{B}\left|R_{z}^{\alpha}(w)\right|^{2} d \mu(w)
$$

for $z \in B$. For $\varphi \in L_{\alpha}^{1}$, we define $\widetilde{\varphi}=\widetilde{\mu}$ where $d \mu=\varphi d V_{\alpha}$.

The notion of Berezin transform can be extended to non-integrable functions which belong to some weighted Lebesgue spaces as follows which is proved in Lemma 3.7 ([4]):

For $\alpha>-1,1 \leq p \leq \infty$ and $\gamma$ real, the Berezin transform is bounded on $L_{\gamma}^{p}$ if and only if $-(n+\alpha+1)<(\gamma+1) / p<\alpha+1$.

Previously, in the holomorphic case on the unit disk, Loaiza, López-García and Pérez-Esteva ([3]) introduced Herz spaces which have mixed norm spaces associated with Schatten classes and they decomposed a given positive Toeplitz operator into a family of local operators and then characterized membership in those spaces. In the harmonic case of unit ball of $\mathbb{R}^{n}$, Choe, Koo and $\mathrm{Na}$ ([2]) showed the boundedness of the Berezin transform on Herz spaces $\mathcal{K}_{q}^{p, \gamma}$ with restricted parameters $p$ and $q$. In the sequel, Choe ([1]), for the full range parameter $q$, proved that the parameter range is a necessary condition for the boundedness of the Berezin transform. Furthermore, he gave the pointwise growth estimate of the Berezin transform on $\mathcal{K}_{q}^{p, \gamma}$.

Also, there is similar result to [2] in the pluriharmonic case of unit ball of $\mathbb{C}^{n}$ that $\mathrm{Na}([4])$ showed as follows:

$$
\begin{aligned}
& \text { For } \alpha>-1,1 \leq p, q \leq \infty \text { and } \gamma \text { real, if } \\
& -(n+\alpha+1)-(\alpha+1) / p<\gamma<(\alpha+1)(1-1 / p), \\
& \text { then the Berezin transform is bounded on } \mathcal{K}_{q}^{p, \gamma} .
\end{aligned}
$$

Motivated by these ideas of [1], we give a more complete result of (1.3) and the growth estimates of the Berezin transforms in the weighted pluriharmonic Bergman space case on the unit ball of $\mathbb{C}^{n}$.

Now, we are about to state our main results as follows.

Theorem 1.1. Let $\alpha>-1,1 \leq p \leq \infty$ and $\gamma$ be real. For $0 \leq q \leq \infty$, the Berezin transform is bounded on $\mathcal{K}_{q}^{p, \gamma}$ if and only if $-(n+\alpha+1)<\gamma+(\alpha+$ 1) $/ p<\alpha+1$ 
Theorem 1.2. Let $\alpha>-1,1 \leq p \leq \infty$ and $\gamma$ be real. Assume $\gamma+(\alpha+$ 1) $/ p \leq \alpha+1$. Then we have the followings: There exist positive constants $C=C(\alpha, p, \gamma)$ such that

(a) if $\gamma+(\alpha+1) / p<\alpha+1$, then $\widetilde{f}(z) \leq C\|f\|_{\mathcal{K}_{\infty}^{p, \alpha} \times}$

$$
\begin{cases}(1-|z|)^{-(n+\alpha+1) / p-\gamma} & \text { if } \gamma>-(n+\alpha+1)(1+1 / p) \\ (1-|z|)^{n+\alpha+1}\left(1+\log \frac{1}{1-|z|}\right) & \text { if } \gamma=-(n+\alpha+1)(1+1 / p) \\ (1-|z|)^{n+\alpha+1} & \text { if } \gamma<-(n+\alpha+1)(1+1 / p)\end{cases}
$$

(b) if $\gamma+(\alpha+1) / p=\alpha+1$, then $\widetilde{f}(z) \leq C\|f\|_{\mathcal{K}_{1}^{p, \alpha}}(1-|z|)^{-(\alpha+1)-n / p}$.

In the next section, for $1 \leq p \leq \infty$, we define the Herz spaces and find all parameters of that spaces that are contained in $L_{\alpha}^{1}$; see (2.3). So, the Berezin transforms are well defined on those spaces. In Section 3, we prove some integral estimates concerning kernel functions and give the estimate of kernel functions in Herz spaces. In Section 4, we establish the condition for the boundedness for the Berezin transforms on Herz spaces. In the last section, we obtain the growth estimates of the Berezin transforms. Theorem 5.2 expresses the growth estimates of the Berezin transforms on such Herz spaces. Similar results on the unit ball of $\mathbb{R}^{n}$ can also be found in [1].

Constants. Throughout the paper, we write $C$ for a various positive constant, which is depend on the given situation. We often abbreviate inessential constants involved in inequalities by writing $X \lesssim Y$ for positive quantities $X$ and $Y$ if the ratio $X / Y$ has a positive upper bound. Also, we write $X \approx Y$ if $X \lesssim Y$ and $Y \lesssim X$.

\section{Herz spaces}

In order to introduce Herz spaces, we let

$$
A_{m}=\left\{z \in B: r_{m} \leq|z|<r_{m+1}\right\},
$$

where $r_{m}=1-2^{-m}$ for each integer $m \geq 0$. We write $\chi_{m}$ for the characteristic function of $A_{m}$ for each $m$. Also, given $\mu \geq 0$, we let $\mu \chi_{m}$ denote the restriction of $\mu$ to $A_{m}$ for each $m$.

Let $\alpha>-1$. Given $\gamma$ real and $0<p, q \leq \infty$, the Herz space $\mathcal{K}_{q}^{p, \gamma}$ is the set of all $L_{\alpha}^{p}$-integrable functions $f$ such that

$$
\|f\|_{\mathcal{K}_{q}^{p, \gamma}}=\left\|\left\{2^{-m \gamma}\left\|f \chi_{m}\right\|_{L_{\alpha}^{p}}\right\}\right\|_{\ell^{q}}<\infty
$$

where $\ell^{q}$ stands for the $q$-summable sequence space. Equipped with the norm above, the space $\mathcal{K}_{q}^{p, \gamma}$ is a Banach space. Also, we let $\mathcal{K}_{0}^{p, \gamma}$ be the subspace of $\mathcal{K}_{\infty}^{p, \gamma}$ consisting of all functions $f \in \mathcal{K}_{\infty}^{p, \gamma}$ such that

$$
\lim _{m \rightarrow \infty} 2^{-m \gamma}\left\|f \chi_{m}\right\|_{L_{\alpha}^{p}}=0 .
$$

Note that $\mathcal{K}_{q}^{p, \gamma} \subset \mathcal{K}_{0}^{p, \gamma}$ for all $q<\infty$. 
In the first place, we recall elementary properties of Herz spaces. For $1 \leq$ $p, q \leq \infty$ and arbitrary real $\gamma$, application of Hölder's inequality yields Hölder's inequality of Herz spaces as follows:

$$
\int_{B} f \bar{g} d V \leq\|f\|_{\mathcal{K}_{q}^{p, \gamma}}\|g\|_{\mathcal{K}_{q^{\prime}}^{p^{\prime},-\gamma}}
$$

for positive measurable functions $f$ and $g$ on $B$; see [2] for details. From now on, $p^{\prime}$ denotes the conjugate exponent of $p$.

We also note that if $1 \leq p \leq \infty$ and if

(2.2) either $\gamma<(\alpha+1)(1-1 / p)$; or $\gamma=(\alpha+1)(1-1 / p)$ and $0<q \leq 1$,

then the Herz space $\mathcal{K}_{q}^{p, \gamma} \subset L_{\alpha}^{1}$; see [4] for details.

In fact we can deduce that

$$
\text { (2.2) if and only if } \mathcal{K}_{q}^{p, \gamma} \subset L_{\alpha}^{1}
$$

for $1 \leq p \leq \infty$. In order to prove this we consider the function $f_{\beta, \delta}$ on $B$ defined by

$$
f_{\beta, \delta}(z)=\frac{1}{(1-|z|)^{\beta}}\left(1+\log \frac{1}{1-|z|}\right)^{-\delta},
$$

where $\beta$ and $\delta$ are given real numbers.

To prove $(2.3)$, we need the following lemma that provides the precise range of parameters for $\mathcal{K}_{q}^{p, \alpha}$ in which the function $f_{\beta, \delta}$ is contained.

Lemma 2.1. $f_{\beta, \delta} \in \mathcal{K}_{q}^{p, \gamma}$ if and only if one of the following conditions holds:

(a) $\gamma+(\alpha+1) / p>\beta$;

(b) $\gamma+(\alpha+1) / p=\beta$ and $\delta>0=q$;

(c) $\gamma+(\alpha+1) / p=\beta$ and $0<1 / \delta<q<\infty$;

(d) $\gamma+(\alpha+1) / p=\beta$ and $\delta \geq 0$ and $q=\infty$.

Proof. For $z \in A_{m}$, note that $f_{\beta, \delta}(z) \approx 2^{m \beta}(1+m)^{-\delta}$ since $1-|z| \approx 2^{-m}$. From this, we have

$$
2^{-m \gamma}\left\|f_{\beta, \delta} \chi_{m}\right\|_{L_{\alpha}^{p}} \approx 2^{-m(\gamma+(\alpha+1) / p-\beta)}(1+m)^{-\delta}
$$

for all $m \geq 0$. Hence we immediately obtain the proof.

Now, we turn to prove the necessary condition of (2.3). Note that for $q<\infty$,

$$
f_{\beta, 0} \in \mathcal{K}_{q}^{p, \gamma} \Longleftrightarrow \beta<\gamma+(\alpha+1) / p
$$

and

$$
f_{\beta, 0} \in \mathcal{K}_{\infty}^{p, \gamma} \Longleftrightarrow \beta \leq \gamma+(\alpha+1) / p .
$$

Since $\left\|f_{\beta, 0}\right\|_{L_{\alpha}^{1}} \approx \int_{0}^{1} t^{-\beta+\alpha} d t$,

$$
f_{\beta, 0} \in L_{\alpha}^{1} \Longleftrightarrow \beta<\alpha+1 .
$$

Then we obtain

$$
f_{\alpha+1,0} \in \mathcal{K}_{q}^{p, \gamma} \quad \text { but } \quad f_{\alpha+1,0} \notin L_{\alpha}^{1}
$$


for all $q$ and $\gamma+(\alpha+1) / p>\alpha+1$. Thus we see that if $\gamma+(\alpha+1) / p>\alpha+1$, then $\mathcal{K}_{q}^{p, \gamma} \not \subset L_{\alpha}^{1}$ for all $q$.

In case $\gamma+(\alpha+1) / p=\alpha+1$, we note that

$$
f_{\alpha+1, \delta} \in L_{\alpha}^{1} \Longleftrightarrow \delta>1
$$

From Lemma 2.1 we obtain for $0<q \leq \infty$ or $q=0$,

$$
f_{\alpha+1,1} \in \mathcal{K}_{q}^{p, \gamma} \quad \text { but } \quad f_{\alpha+1,1} \notin L_{\alpha}^{1} .
$$

Thus (2.3) is proved.

\section{Integral estimates}

To prove our main result Theorem 4.1, we begin with recalling some integral estimates relevant to the kernel functions; see Proposition 1.4.10 of [5]. Here $d S$ is the surface area measure on $\partial B$, the boundary of $B$.

Lemma 3.1. For $-1<a<\infty$, c real and $z \in B$,

$$
\begin{aligned}
\int_{\partial B} \frac{d S(\zeta)}{|1-z \cdot \bar{\zeta}|^{n+c}} & \approx \int_{B} \frac{(1-|w|)^{a}}{|1-z \cdot \bar{w}|^{n+1+a+c}} d V(w) \\
& \approx \begin{cases}1 & \text { if } c<0 \\
1-\log \left(1-|z|^{2}\right) & \text { if } c=0 \\
\left(1-|z|^{2}\right)^{-c} & \text { if } c>0 .\end{cases}
\end{aligned}
$$

The constants suppressed above depend only on $n$ and $c$.

Let $\alpha>-1$. We need to estimate two types of integrals $J_{c}$ and $I_{a, c}$ defined for given $-1<a<\infty$ and $c$ real as follows:

$$
J_{c}(z)=\int_{\partial B}\left|R_{z}^{\alpha}(\zeta)\right|^{1+\frac{c-1}{n+\alpha+1}} d S(\zeta)
$$

and

$$
I_{a, c}(z)=\int_{B}\left|R_{z}^{\alpha}(w)\right|^{1+\frac{a+c}{n+\alpha+1}}\left(1-|w|^{2}\right)^{a} d V(w)
$$

for $z \in B$.

We now estimate of $J_{c}(z)$.

Proposition 3.2. Let $\alpha>-1$. For $c$ real and $z \in B$,

$$
J_{c}(z) \approx \begin{cases}1 & \text { if } \alpha+c<0 \\ 1-\log \left(1-|z|^{2}\right) & \text { if } \alpha+c=0 \\ \left(1-|z|^{2}\right)^{-(\alpha+c)} & \text { if } \alpha+c>0\end{cases}
$$

The constants suppressed above depend only on $n, \alpha$ and $c$. 
Proof. From Lemma 3.1, we obtain the upper estimates easily. Thus, we only prove the lower estimates. To do this, we consider two subsets of $\partial B$ as follows: for $z \in B$,

$$
\Gamma_{z}=\left\{\zeta \in \partial B:|\arg (z \cdot \bar{\zeta})|<\frac{\pi}{4(n+\alpha+1)} \quad \text { and } \quad|1-z \cdot \bar{\zeta}| \leq 1\right\}
$$

and

$$
\Lambda_{z}=\left\{\zeta \in \partial B:|\arg (1-z \cdot \bar{\zeta})|>\frac{\pi}{3(n+\alpha+1)}+\epsilon \quad \text { and } \quad|1-z \cdot \bar{\zeta}|>1\right\}
$$

for sufficiently small $\epsilon>0$. Write $1-z \cdot \bar{\zeta}=r e^{i \theta}$. If $|z| \leq \frac{1}{2}$, then we have for any $\zeta \in \Gamma_{z}$

$$
2 \cos (n+\alpha+1) \theta-r^{n+\alpha+1} \gtrsim 1
$$

and

$$
J_{c}(z) \gtrsim \int_{\Gamma_{z}}|1-z \cdot \bar{\zeta}|^{-(n+\alpha+c)} d S(\zeta)
$$

In case $\alpha+c \geq 0$, we obtain

$$
\int_{\Gamma_{z}}|1-z \cdot \bar{\zeta}|^{-(n+\alpha+c)} d S(\zeta) \gtrsim 1
$$

On the other case $\alpha+c<0$, we have the estimate

$$
\int_{\Gamma_{z}} \frac{d S(\zeta)}{|1-z \cdot \bar{\zeta}|^{n+\alpha+c}}=\int_{\Gamma_{z}} \frac{|1-z \cdot \bar{\zeta}|^{-2(\alpha+c)}}{|1-z \cdot \bar{\zeta}|^{n-(\alpha+c)}} d S(\zeta) \gtrsim(1-|z|)^{-2(\alpha+c)} \gtrsim 1 .
$$

If $|z| \geq \frac{1}{2}$, then we consider the set $\Lambda_{z}$. For any $\zeta \in \Lambda_{z}$, we have

$$
r^{n+\alpha+1}-2 \cos (n+\alpha+1) \theta \gtrsim 1
$$

and

$$
\begin{aligned}
J_{c}(z) & \gtrsim \int_{\Lambda_{z}}|1-z \cdot \bar{\zeta}|^{-(n+\alpha+c)} d S(\zeta) \\
& \geq \int_{\left\{\zeta \in \partial B: \frac{\pi}{2}<\arg (z \cdot \bar{\zeta})<\frac{\pi}{2}+\delta\right\}}|1-z \cdot \bar{\zeta}|^{-(n+\alpha+c)} d S(\zeta)
\end{aligned}
$$

for some constant $\delta>0$ which is independent of $z$. Let $\lambda=\frac{n+\alpha+c}{2}$. For $|x|<1$, we consider the binomial series

$$
(1-x)^{\lambda}=\sum_{k=0}^{\infty} \frac{\Gamma(k+\lambda)}{k ! \Gamma(\lambda)} x^{k} .
$$

We assume $z=|z| e_{1}$. By using (3.2) and orthogonality of $\left\{(z \cdot \bar{\zeta})^{m}\right\}$, we obtain

$$
\begin{aligned}
\int_{G}|1-z \cdot \bar{\zeta}|^{-(n+\alpha+c)} d S(\zeta) & =\left.\sum_{k=0}^{\infty} \int_{G} \frac{\mid \Gamma(k+\lambda)}{k ! \Gamma(\lambda)}\left(|z| \zeta_{1}\right)^{k}\right|^{2} d S(\zeta) \\
& =\sum_{k=0}^{\infty} \frac{\Gamma^{2}(k+\lambda)}{(k !)^{2} \Gamma^{2}(\lambda)}|z|^{2 k} \int_{G}\left|\zeta_{1}\right|^{2 k} d S(\zeta)
\end{aligned}
$$


Here $G=\left\{\zeta \in \partial B: \frac{\pi}{2}<\arg \left(\zeta_{1}\right)<\frac{\pi}{2}+\delta\right\}$. To calculate above last integral we claim that

$$
\int_{G}\left|\zeta_{1}\right|^{2 k} d S(\zeta) \approx \int_{\partial B}\left|\zeta_{1}\right|^{2 k} d S(\zeta)
$$

for nonnegative $k$. To prove (3.3), we put $\zeta_{1}^{\prime}=e^{i \phi} \zeta_{1}$ and $\zeta_{i}^{\prime}=\zeta_{i}$ for any fixed angle $\phi$ and $i=2,3, \ldots, n$. Since absolute value of the Jacobian of this mapping is 1 , we have

$$
\begin{aligned}
\int_{G}\left|\zeta_{1}\right|^{2 k} d S(\zeta) & =\int_{\left\{\zeta^{\prime} \in \partial B: \frac{\pi}{2}<\arg \left(e^{-i \phi} \zeta_{1}^{\prime}\right)<\frac{\pi}{2}+\delta\right\}}\left|\zeta_{1}^{\prime}\right|^{2 k} d S\left(\zeta^{\prime}\right) \\
& =\int_{\left\{\zeta \in \partial B: \frac{\pi}{2}+\phi<\arg \left(\zeta_{1}\right)<\frac{\pi}{2}+\delta+\phi\right\}}\left|\zeta_{1}\right|^{2 k} d S(\zeta) .
\end{aligned}
$$

We write

$$
\int_{\partial B}\left|\zeta_{1}\right|^{2 k} d S(\zeta)=\sum_{i} \int_{\left\{\zeta \in \partial B: \frac{\pi}{2}+\delta(i-1)<\arg \left(\zeta_{1}\right)<\frac{\pi}{2}+\delta i\right\}}\left|\zeta_{1}\right|^{2 k} d S(\zeta),
$$

which proves (3.3). Thus, using (3.3) with Proposition 1.4.9 of [5], we have the following estimate

$$
J_{c}(z) \gtrsim \frac{\Gamma(n)}{\Gamma^{2}(\lambda)} \sum_{k=0}^{\infty} \frac{\Gamma^{2}(k+\lambda)}{\Gamma(k+1) \Gamma(k+n)}|z|^{2 k} .
$$

By Stirling's formula, we see that the coefficients in this last series are of order $k^{\alpha+c-1}$ as $k \rightarrow \infty$. This proves the assertions.

Lemma 3.3. Let $\alpha>-1$. For $-1<a<\infty$, c real and $z \in B$,

$$
I_{a, c}(z) \approx \begin{cases}1 & \text { if } \alpha+c<0 \\ 1-\log \left(1-|z|^{2}\right) & \text { if } \alpha+c=0 \\ \left(1-|z|^{2}\right)^{-(\alpha+c)} & \text { if } \alpha+c>0 .\end{cases}
$$

The constants suppressed above depend only on $n, \alpha$ and $c$.

Proof. One can easily prove the upper estimates by Lemma 3.1. So we now prove the lower estimates. By using integration in polar coordinates and (1.1), we obtain

$$
\begin{aligned}
I_{a, c}(z) & =\int_{0}^{1} r^{2 n-1}\left(1-r^{2}\right)^{a} \int_{\partial B}\left|R_{z}^{\alpha}(r \zeta)\right|^{1+\frac{a+c}{n+\alpha+1}} d S(\zeta) d r \\
& =\int_{0}^{1} r^{2 n-1}\left(1-r^{2}\right)^{a} \int_{\partial B}\left|R_{r z}^{\alpha}(\zeta)\right|^{1+\frac{a+c}{n+\alpha+1}} d S(\zeta) d r .
\end{aligned}
$$

If $|r z| \leq 1 / 2$, then we obtain the following

$$
I_{a, c}(z) \gtrsim \int_{0}^{1} r^{2 n-1}\left(1-r^{2}\right)^{a} d r \gtrsim 1
$$


In the other case $|r z|>1 / 2$, we consider the subset $\Gamma_{z}$ of $\partial B$ as in Proposition 3.2. Thus, (3.3) and Proposition 3.2 imply that, for $|r z|>1 / 2$,

$$
\begin{aligned}
& I_{a, c}(z) \\
\geq & \int_{0}^{1} r^{2 n-1}\left(1-r^{2}\right)^{a} \int_{\Gamma_{r z}} \frac{1}{|1-r z \cdot \bar{w}|^{n+\alpha+1+a+c}} d S(\zeta) d r \\
\gtrsim & \int_{0}^{1} r^{2 n-1}\left(1-r^{2}\right)^{a} \int_{\left\{\zeta \in \partial B \mid \frac{\pi}{2}<\arg (r z \cdot \bar{w})<\frac{\pi}{2}+\delta\right\}} \frac{1}{|1-r z \cdot \bar{w}|^{n+\alpha+1+a+c}} d S(\zeta) d r \\
\approx & \int_{0}^{1} r^{2 n-1}\left(1-r^{2}\right)^{a} \int_{\partial B} \frac{1}{|1-r z \cdot \bar{w}|^{n+\alpha+1+a+c}} d S(\zeta) d r \\
= & \int_{B} \frac{(1-|w|)^{a}}{|1-z \cdot \bar{w}|^{n+1+a+\alpha+c}} d V(w) .
\end{aligned}
$$

The proof is complete by Lemma 3.1.

The following result presents the estimate of kernel functions in Herz spaces.

Lemma 3.4. Let $\alpha>-1,1 \leq p, q \leq \infty$ and assume $-(\alpha+1) / p<\gamma<$ $2(n+\alpha+1)-(n+\alpha+1) / p$. Then there exists a constant $C=C(\alpha, p, q, \gamma)$ such that

for $z \in B$.

$$
\left\|\left(R_{z}^{\alpha}\right)^{2}\right\|_{\mathcal{K}_{q}^{p, \gamma}} \leq \frac{C}{(1-|z|)^{2(n+\alpha+1)-(n+\alpha+1) / p-\gamma}}
$$

Proof. Fix $z \in B$. Note that for $c>0$,

$$
\int_{\partial B} \frac{d S(\zeta)}{|1-z \cdot \bar{\zeta}|^{n+c}} \approx\left(1-|z|^{2}\right)^{-c} .
$$

We first estimate $\left\|\left(R_{z}^{\alpha}\right)^{2} \chi_{m}\right\|_{L_{\alpha}^{p}}$. Thus, for $p<\infty$, (1.2) yields

$$
\begin{aligned}
\left\|\left(R_{z}^{\alpha}\right)^{2} \chi_{m}\right\|_{L_{\alpha}^{p}}^{p} & \lesssim \int_{A_{m}}|1-z \cdot \bar{w}|^{-2(n+\alpha+1) p} 2^{-m \alpha} d V(w) \\
& =2^{-m \alpha} \int_{r_{m}}^{r_{m+1}} r^{2 n-1} \int_{\partial B}|1-r z \cdot \bar{\zeta}|^{-2(n+\alpha+1) p} d S(\zeta) d r \\
& \lesssim 2^{-m \alpha} \int_{r_{m}}^{r_{m+1}}\left(1-|r z|^{2}\right)^{-2 p(n+\alpha+1)+n} d r \\
& \lesssim \frac{\left(r_{m+1}-r_{m}\right) 2^{-m \alpha}}{\left(1-r_{m+1}|z|\right)^{2 p(n+\alpha+1)-n}} \\
& \lesssim \frac{2^{-m(\alpha+1)}}{\left(1-|z|+2^{-m}|z|\right)^{2 p(n+\alpha+1)-n}}
\end{aligned}
$$

We therefore conclude

$$
\left\|\left(R_{z}^{\alpha}\right)^{2} \chi_{m}\right\|_{L_{\alpha}^{p}} \lesssim \frac{2^{-m(\alpha+1) / p}}{\left(1-|z|+2^{-m}|z|\right)^{2(n+\alpha+1)-n / p}}
$$


and this estimate is uniform in $m$. Also, this estimate remains valid for $p=\infty$, because $\left|R_{z}^{\alpha}(w)\right| \lesssim(1-|z||w|)^{-(n+\alpha+1)}$.

Now, we estimate the Herz norm of $\left(R_{z}^{\alpha}\right)^{2}$. Let $1 \leq q \leq \infty$ and $|z| \leq 1 / 2$. Using (3.4), it is easy to see that

$$
\left\|\left(R_{z}^{\alpha}\right)^{2}\right\|_{\mathcal{K}_{q}^{p, \gamma}} \lesssim\left\|\left\{2^{-m(\gamma+(\alpha+1) / p)}\right\}\right\|_{\ell^{q}} \approx 1 .
$$

Consider the case $|z|>1 / 2$. First, if $(1-|z|) \leq 2^{-m}$, then we have by (3.4)

$$
\begin{aligned}
2^{-m \gamma}\left\|\left(R_{z}^{\alpha}\right)^{2} \chi_{m}\right\|_{L_{\alpha}^{p}} & \lesssim \frac{2^{-m(\gamma+(\alpha+1) / p)}}{2^{-m(2(n+\alpha+1)-n / p)}} \\
& \lesssim \frac{1}{(1-|z|)^{2(n+\alpha+1)-(n+\alpha+1) / p-\gamma}} .
\end{aligned}
$$

Meanwhile, if $(1-|z|)>2^{-m}$, then we have by $(3.4)$

$$
\begin{aligned}
2^{-m \gamma}\left\|\left(R_{z}^{\alpha}\right)^{2} \chi_{m}\right\|_{L_{\alpha}^{p}} & \lesssim \frac{2^{-m(\gamma+(\alpha+1) / p)}}{(1-|z|)^{2(n+\alpha+1)-n / p}} \\
& <\frac{1}{(1-|z|)^{2(n+\alpha+1)-(n+\alpha+1) / p-\gamma}} .
\end{aligned}
$$

Then the case $q=\infty$ follows. Also, for $q<\infty$, it follows from (3.5) and (3.6) that

$$
\begin{aligned}
\left\|\left(R_{z}^{\alpha}\right)^{2}\right\|_{\mathcal{K}_{q}^{p, \gamma}}^{q} & =\sum_{m} 2^{-m q \gamma}\left\|\left(R_{z}^{\alpha}\right)^{2} \chi_{m}\right\|_{L_{\alpha}^{p}}^{q} \\
& =\sum_{m \leq \log _{2}(1-|z|)^{-1}}+\sum_{m>\log _{2}(1-|z|)^{-1}} \\
& \lesssim \frac{1}{(1-|z|)^{q(2(n+\alpha+1)-(n+\alpha+1) / p-\gamma)}},
\end{aligned}
$$

as desired. The proof is complete.

Lemma 3.5. Let $A=\{z \in B: 0 \leq a \leq|z|<b \leq 1\}$ be an annulus in $B$. Given $\alpha+c>0$, there exists a positive constant $C=C(n, \alpha, c)$ such that

$$
\frac{C^{-1}}{\left(1-a^{2}|z|^{2}\right)^{\alpha+c}} \leq \frac{1}{b-a} \int_{A}\left|R_{z}^{\alpha}(w)\right|^{1+\frac{c-1}{n+\alpha+1}} d V(w) \leq \frac{C}{\left(1-b^{2}|z|^{2}\right)^{\alpha+c}}
$$

for $z \in B$.

Proof. Let $z \in B$. By integration in polar coordinates and Proposition 3.2, we have

$$
\begin{aligned}
\int_{A}\left|R_{z}^{\alpha}(w)\right|^{1+\frac{c-1}{n+\alpha+1}} d V(w) & =\int_{a}^{b} r^{2 n-1} \int_{\partial B}\left|R_{r z}^{\alpha}(\zeta)\right|^{1+\frac{c-1}{n+\alpha+1}} d S(\zeta) d r \\
& \approx \int_{a}^{b} r^{2 n-1} \frac{1}{\left(1-r^{2}|z|^{2}\right)^{\alpha+c}} d r
\end{aligned}
$$

which implies the lemma. 


\section{Boundedness}

In this section, we formulate one of our main results.

Theorem 4.1. Let $\alpha>-1,1 \leq p \leq \infty$ and $\gamma$ be real. For $0 \leq q \leq \infty$, the Berezin transform is bounded on $\mathcal{K}_{q}^{p, \bar{\gamma}}$ if and only if $-(n+\alpha+\overline{1})<\gamma+(\alpha+$ 1) $/ p<\alpha+1$.

The sufficiency of Theorem 4.1 follows from Lemma 4.5 of [4]. In order to prove the necessity of Theorem 4.1, we need to prove: Given $0 \leq q \leq \infty$ there exists some $f \in \mathcal{K}_{q}^{p, \gamma}$ but $\tilde{f} \notin \mathcal{K}_{q}^{p, \gamma}$.

Throughout this section we consider parameters $\alpha>-1,0<p \leq \infty$ and $\gamma$ real such that

(4.1) either $\gamma+(\alpha+1) / p \leq-(n+\alpha+1) ; \quad$ or $\quad \gamma+(\alpha+1) / p \geq \alpha+1$.

We will prove this for general $p$, which is not necessarily greater than or equal to 1.

Given $\beta$ and $\delta$ real, let

$$
h_{\beta, \delta}(r)=\frac{1}{(1-r)^{\beta}}\left(1+\log \frac{1}{1-r}\right)^{-\delta}, \quad 0 \leq r<1
$$

so that

$$
f_{\beta, \delta}(z)=h_{\beta, \delta}(|z|), \quad z \in B .
$$

We separately consider two cases in (4.1) for convenience.

4.1. The case $\gamma+(\alpha+1) / p \leq-(n+\alpha+1)$ : We further split this case into the following four cases:

(1) $\gamma+(\alpha+1) / p<-(n+\alpha+1)$ with $q$ arbitrary;

(2) $\gamma+(\alpha+1) / p=-(n+\alpha+1)$ with $q=0$;

(3) $\gamma+(\alpha+1) / p=-(n+\alpha+1)$ with $0<q<\infty$;

(4) $\gamma+(\alpha+1) / p=-(n+\alpha+1)$ with $q=\infty$.

Example 1. If (1) or (2) holds, then $\tilde{\chi}_{A} \notin \mathcal{K}_{q}^{p, \gamma}$ for any compact annular region $A \subset B$.

Proof. Assume that (1) or (2) holds and let $A \subset B$ be an arbitrary compact annular region. By Lemma 3.5 we have

$$
\tilde{\chi}_{A} \gtrsim(1-|z|)^{n+\alpha+1}=f_{-(n+\alpha+1), 0}(z)
$$

for $z \in B$. Using Lemma 2.1, we have $f_{-(n+\alpha+1), 0} \notin \mathcal{K}_{q}^{p, \gamma}$ from the condition (1) or (2) and thus $\tilde{\chi}_{A} \notin \mathcal{K}_{q}^{p, \gamma}$.

Example 2. If (3) holds, then $f_{-(n+\alpha+1), \delta} \in \mathcal{K}_{q}^{p, \gamma}$, but $\tilde{f}_{-(n+\alpha+1), \delta} \notin \mathcal{K}_{q}^{p, \gamma}$ for $\delta>1$ with $\delta>1 / q$.

Proof. Assume that (3) holds. Let $\delta>1$ and $\delta>1 / q$. Then we have $f_{-(n+\alpha+1), \delta} \in \mathcal{K}_{q}^{p, \gamma}$ by Lemma 2.1 . 
Now, we estimate $\widetilde{f}_{-(n+\alpha+1), \delta}$. Let $z \in B$ and assume $|z| \geq 1 / 2$. Note that

$$
1-r^{2}|z|^{2} \approx 1-r|z|=(1-|z|)+|z|(1-r) \approx(1-|z|)+(1-r)
$$

for $|z| \geq 1 / 2$ and $0 \leq r<1$. By combining this with (1.2), integration in polar coordinates and Proposition 3.2, we have

$$
\begin{aligned}
& \widetilde{f}_{-(n+\alpha+1), \delta}(z) \\
\approx & (1-|z|)^{n+\alpha+1} \int_{0}^{1} r^{2 n-1}\left(1-r^{2}\right)^{\alpha} \int_{\partial B}\left|R_{r z}^{\alpha}(\zeta)\right|^{2} h_{-(n+\alpha+1), \delta}(r) d S(\zeta) d r \\
\approx & (1-|z|)^{n+\alpha+1} \int_{0}^{1} \frac{r^{2 n-1}\left(1-r^{2}\right)^{\alpha} h_{-(n+\alpha+1), \delta}(r)}{\left(1-r^{2}|z|^{2}\right)^{n+2(\alpha+1)}} d r \\
\geq & (1-|z|)^{n+\alpha+1} \int_{\frac{1}{2}}^{1} \frac{\left(1-r^{2}\right)^{\alpha} h_{-(n+\alpha+1), \delta}(r)}{[(1-|z|)+(1-r)]^{n+2(\alpha+1)}} d r \\
\gtrsim & (1-|z|)^{n+\alpha+1} \int_{\frac{1}{2}}^{1} \frac{(1-\log (1-r))^{-\delta}}{1-r} d r \\
\approx & (1-|z|)^{n+\alpha+1}
\end{aligned}
$$

for $|z| \geq 1 / 2$. Thus we obtain for $z \in B$,

$$
\tilde{f}_{-(n+\alpha+1), \delta}(z) \gtrsim f_{-(n+\alpha+1), 0}(z)
$$

so that $\tilde{f}_{-(n+\alpha+1), \delta} \notin \mathcal{K}_{q}^{p, \gamma}$ by $(3)$ and Lemma 2.1 .

Example 3. If (4) holds, then $f_{-(n+\alpha+1), 0} \in \mathcal{K}_{\infty}^{p, \gamma}$, but $\widetilde{f}_{-(n+\alpha+1), 0} \notin \mathcal{K}_{\infty}^{p, \gamma}$.

Proof. Assume that (4) holds. Then we have $f_{-(n+\alpha+1), 0} \in \mathcal{K}_{\infty}^{p, \gamma}$ by Lemma 2.1. Also, we have by the same manner of the proof of Example 2

$$
\begin{aligned}
\tilde{f}_{-(n+\alpha+1), 0}(z) & \approx(1-|z|)^{n+\alpha+1} \int_{0}^{1} \frac{r^{2 n-1}\left(1-r^{2}\right)^{\alpha} h_{-(n+\alpha+1), 0}(r)}{\left(1-r^{2}|z|^{2}\right)^{n+2(\alpha+1)}} d r \\
& =(1-|z|)^{n+\alpha+1} \int_{0}^{1} \frac{r^{2 n-1}\left(1-r^{2}\right)^{n+2 \alpha+1}}{\left(1-r^{2}|z|^{2}\right)^{n+2(\alpha+1)}} d r \\
& \gtrsim(1-|z|)^{n+\alpha+1} \int_{\frac{1}{2}}^{1} \frac{d r}{1-r} \\
& =\infty
\end{aligned}
$$

for $z \in B$. Thus we have the desired result.

4.2. The case $\gamma+(\alpha+1) / p \geq \alpha+1$ : We further split this case into the following three cases:

(5) $\gamma+(\alpha+1) / p>\alpha+1$;

(6) $\gamma+(\alpha+1) / p=\alpha+1$, with $1<q \leq \infty$ or $q=0$;

(7) $\gamma+(\alpha+1) / p=\alpha+1$, with $0<q \leq 1$.

The next example covers the subcases (5) and (6). 
Example 4. If (5) or (6) holds, then $f_{\alpha+1,1} \in \mathcal{K}_{q}^{p, \gamma}$ but $\widetilde{f}_{\alpha+1,1}=\infty$ on $B$.

Proof. Under the condition (5) or (6), we have $f_{\alpha+1,1} \in \mathcal{K}_{q}^{p, \gamma}$ by Lemma 2.1. Note that $h_{\alpha+1,1}$ is not integrable near $r=1$. Thus, following the proof of Example 2, we have by Proposition 3.2

$$
\begin{aligned}
\widetilde{f}_{\alpha+1,1}(z) & \approx(1-|z|)^{n+\alpha+1} \int_{0}^{1} \frac{r^{2 n-1}\left(1-r^{2}\right)^{\alpha} h_{\alpha+1,1}(r)}{\left(1-r^{2}|z|^{2}\right)^{n+2(\alpha+1)}} d r \\
& \gtrsim(1-|z|)^{n+\alpha+1} \int_{\frac{1}{2}}^{1} \frac{d r}{(1-r)^{n+2 \alpha+3}(1-\log (1-r))} \\
& =(1-|z|)^{n+\alpha+1} \int_{0}^{\frac{1}{2}} \frac{d t}{t^{n+2 \alpha+3}(1-\log t)} \\
& =\infty
\end{aligned}
$$

for all $z \in B$, as desired.

In case of (7), we have $\mathcal{K}_{q}^{p, \gamma} \subset L_{\alpha}^{1}$ by (2.3) so that Berezin transform is well defined on that space.

Example 5. If (7) holds, then $f_{\alpha+1, \delta} \in \mathcal{K}_{q}^{p, \gamma}$ but $\tilde{f}_{\alpha+1, \delta} \notin \mathcal{K}_{q}^{p, \gamma}$ for all $\delta>1$ with $\delta>1 / q$.

Proof. Assume that (7) holds. Let $\delta>1$ and $\delta>1 / q$. Since $\delta>1 / q$, we have $f_{\alpha+1, \delta} \in \mathcal{K}_{q}^{p, \gamma}$ by Lemma 2.1 .

Now, we estimate $\widetilde{f}_{\alpha+1, \delta}$. Let $z \in B$ and assume $|z| \geq 1 / 2$. Following the proof of Example 2, we have

$$
\begin{aligned}
\widetilde{f}_{\alpha+1, \delta}(z) & \approx(1-|z|)^{n+\alpha+1} \int_{0}^{1} \frac{r^{2 n-1}\left(1-r^{2}\right)^{\alpha} h_{\alpha+1, \delta}(r)}{\left(1-r^{2}|z|^{2}\right)^{n+2(\alpha+1)}} d r \\
& \gtrsim(1-|z|)^{n+\alpha+1} \int_{\frac{1}{2}}^{1} \frac{d r}{(1-r)^{n+2 \alpha+3} \log (1-(1-r))^{\delta}} \\
& =\infty
\end{aligned}
$$

for $|z| \geq 1 / 2$ and thus for all $z \in B$.

\section{Growth estimates}

Finally, we deal with the pointwise growth estimates of the Berezin transforms on Herz spaces. Before doing this, we first described that parameters $p, q, \alpha$ and $\gamma$ satisfy (2.3) so that the Berezin transform is well defined on Herz spaces with such parameters. In this section we show pointwise growth estimates of Berezin transforms in the following two cases:

(i) $\gamma+(\alpha+1) / p<\alpha+1$ and $q=1$;

(ii) $\gamma+(\alpha+1) / p=\alpha+1$ and $q=\infty$. 
Given a nonnegative measurable function $f$ on $B$, we note that (2.1) implies

$$
\widetilde{f}(z) \lesssim(1-|z|)^{n+\alpha+1}\|f\|_{\mathcal{K}_{q}^{p, \gamma}}\left\|\left(R_{z}^{\alpha}\right)^{2}\right\|_{\mathcal{K}_{q^{\prime}}^{p^{\prime},-\gamma}}
$$

for $z \in B$ and $1 \leq q \leq \infty$. So, we first examine the growth estimate of Herz norms of $R_{z}^{2}$.

Lemma 5.1. Let $\alpha>-1,1 \leq p \leq \infty$ and $\gamma$ be real. Assume $\gamma+(\alpha+1) / p \geq$ 0 . Then there exist positive constants $C=C(\alpha, p, \gamma)$ such that the following inequalities holds: For $z \in B$,

(a) if $\gamma+(\alpha+1) / p>0$, then $\left\|\left(R_{z}^{\alpha}\right)^{2}\right\|_{\mathcal{K}_{1}^{p, \gamma}} \leq C \times$

$$
\begin{cases}1 & \text { if } \gamma>(n+\alpha+1)(2-1 / p) \\ 1+\log \frac{1}{1-|z|} & \text { if } \gamma=(n+\alpha+1)(2-1 / p) \\ (1-|z|)^{-2(n+\alpha+1)+(n+\alpha+1) / p+\gamma} & \text { if } \gamma<(n+\alpha+1)(2-1 / p) ;\end{cases}
$$

(b) if $\gamma+(\alpha+1) / p=0$, then $\left\|\left(R_{z}^{\alpha}\right)^{2}\right\|_{\mathcal{K}_{\infty}^{p, \gamma}} \leq C(1-|z|)^{-2(n+\alpha+1)+n / p}$.

Proof. Fix $z \in B$. Let $1 \leq p \leq \infty$. If $\gamma+(\alpha+1) / p=0$, then (b) immediately holds from the proof of Lemma 3.4. We now turn to the case $\gamma+(\alpha+1) / p>0$. Using (3.5), (3.6) and (3.7), we have

$$
\begin{aligned}
\left\|\left(R_{z}^{\alpha}\right)^{2}\right\|_{\mathcal{K}_{1}^{p, \gamma}} \lesssim & \sum_{m \leq \log _{2}(1-|z|)^{-1}} 2^{m(2(n+\alpha+1)-(n+\alpha+1) / p-\gamma)} \\
& +\frac{1}{(1-|z|)^{2(n+\alpha+1)-n / p}} \sum_{m>\log _{2}(1-|z|)^{-1}} 2^{-m(\gamma+(\alpha+1) / p)} \\
:=I & +I I .
\end{aligned}
$$

One can verify the following estimate by some calculation:

$$
I \approx \begin{cases}1 & \text { if } \gamma>(n+\alpha+1)(2-1 / p) \\ 1+\log \frac{1}{1-|z|} & \text { if } \gamma=(n+\alpha+1)(2-1 / p) \\ (1-|z|)^{-2(n+\alpha+1)+(n+\alpha+1) / p+\gamma} & \text { if } \gamma<(n+\alpha+1)(2-1 / p) .\end{cases}
$$

Also, since $\gamma+(\alpha+1) / p>0$, we have

$$
\sum_{m>\log _{2}(1-|z|)^{-1}} 2^{-m(\gamma+(\alpha+1) / p)} \approx(1-|z|)^{\gamma+(\alpha+1) / p}
$$

so that $I I \approx(1-|z|)^{-2(n+\alpha+1)+(n+\alpha+1) / p+\gamma}$. Combining these two estimates, we can prove (a).

The following is our second main result.

Theorem 5.2. Let $\alpha>-1,1 \leq p \leq \infty$ and $\gamma$ be real. Assume $\gamma+(\alpha+$ $1) / p \leq \alpha+1$. Then we have the followings: There exist positive constants $C=C(\alpha, p, \gamma)$ such that 
(a) if $\gamma+(\alpha+1) / p<\alpha+1$, then $\tilde{f}(z) \leq C\|f\|_{\mathcal{K}_{\infty}^{p, \alpha} \times}$

$$
\begin{cases}(1-|z|)^{-(n+\alpha+1) / p-\gamma} & \text { if } \gamma>-(n+\alpha+1)(1+1 / p) \\ (1-|z|)^{n+\alpha+1}\left(1+\log \frac{1}{1-|z|}\right) & \text { if } \gamma=-(n+\alpha+1)(1+1 / p) \\ (1-|z|)^{n+\alpha+1} & \text { if } \gamma<-(n+\alpha+1)(1+1 / p)\end{cases}
$$

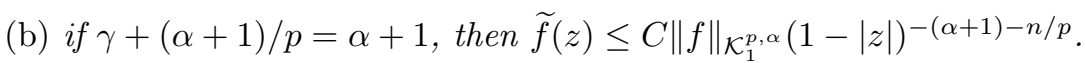

Proof. This follows from (5.1) and Lemma 5.1.

\section{References}

[1] B. R. Choe, Note on the Berezin transform on Herz spaces, RIMS Kyokuroku 1519 (2006), 21-37.

[2] B. R. Choe, H. Koo, and K. Na, Positive Toeplitz operators of Schatten-Herz type, Nagoya Math. J. 185 (2007), 31-62.

[3] M. Loaiza, M. López-García, and S. Pérez-Esteva, Herz classes and Toeplitz operators in the disk, Integral Equations Operator Theory 53 (2005), no. 2, 287-296.

[4] K. Na, Positive Schatten(-Herz) class Toeplitz operators on pluriharmonic Bergman spaces, Integral Equations Operator Theory 64 (2009), no. 3, 409-428.

[5] W. Rudin, Function Theory in the Unit Ball of $C^{n}$, Springer Verlag, 1980.

Chu-Hee Cho

School of Mathematical Sciences

Seoul National University

SEOUl 151-742, Korea

E-mail address: akilus@snu.ac.kr

KYUNGUK NA

General Education, Mathematics

HANSHIN UNIVERSITY

GyeOngGi 447-791, Korea

E-mail address: nakyunguk@hs.ac.kr 